\title{
El conocimiento generado a partir de la aplicación de un proceso de diagnóstico por estudiantes normalistas en escuelas de educación primaria: la relación práctica profesional e investigación educativa
}

\section{The knowledge generated from the application of a diagnostic process by normalist students in elementary schools: the professional practical relationship and educational research}

\author{
CHAPA-CHAPA, Mireya*† \\ Escuela Normal Pablo Livas. Dr. Coss 500 CP 64200. Sabinas Hidalgo, N.L., México.
}

ID $1^{\text {er }}$ Autor: Mireya, Chapa-Chapa

DOI: $10.35429 / J U P .2020 .11 .4 .24 .33$

Recibido: 25 de Enero, 2020; Aceptado: 30 de Junio, 2020

\begin{abstract}
Resumen
La transformación de las escuelas normales en instituciones de educación superior en las que la investigación educativa sea una actividad sustantiva es una de las exigencias actuales a la formación de profesores de educación básica (INEE, 2015). Este trabajo representa un esfuerzo inicial por hacer realidad esta premisa y en él se dan a conocer los resultados de la sistematización y análisis del proceso de diagnóstico realizado como parte del curso Trabajo docente e innovación, que forma parte del $5^{\circ}$ semestre de la Licenciatura en Educación Básica. Este proceso generó conocimientos en dos sentidos: las competencias puestas en juego por los docentes en formación, así como la obtención de un panorama general de la situación de 24 grupos de educación primaria en aspectos como competencia lectora y matemáticas. El estudio se sustenta en una revisión de literatura que considera el trayecto formativo de práctica profesional, los conceptos de diagnóstico e innovación y el modelo educativo del Plan de estudios 2012 para la formación de maestros de educación primaria. A partir de metodología cualitativa para el análisis de los resultados, se concluye que la investigación educativa tiene un papel importante como estrategia para la mejora, abre la posibilidad de iniciar una nueva etapa en la historia de la formación docente en México.
\end{abstract}

Formación inicial docente, Investigación educativa

\begin{abstract}
The transformation of mexican escuelas normales in institutions of higher education in which educational research is a substantive activity is one of the current requirements to the formation of elementary education teachers (INEE, 2015). This study represents an initial effort to make truth this premise and describes the results of a systematization and analysis process of the diagnostic process made as part of the course Trabajo docente e innovación, which is part of the $5^{\circ}$ semester of the Bachelor degree of Elementary Education. This process generated knowledge in two ways: the skills brought into play by student teachers as well as obtaining an overview of the status of 24 groups of elementary education in reading and problem solving, among others. The study is based on a literature review that considers the training course of professional practice in teacher education, diagnostic and innovation concepts and the educational model of the Plan 2012 for the initial training of elementary school teachers. From qualitative methodology for analyzing the results, the study concludes with the idea that educational research has an important role as a strategy for improvement and opens the possibility of starting a new era in the history of teacher education in Mexico.
\end{abstract}

Initial teaching studies, Educational research

Citación: CHAPA-CHAPA, Mireya. El conocimiento generado a partir de la aplicación de un proceso de diagnóstico por estudiantes normalistas en escuelas de educación primaria: la relación práctica profesional e investigación educativa. Revista de Políticas Universitarias. 2020. 4-11:24-33.

\footnotetext{
* Correspondencia del Autor (Correo electrónico: mirechapa@ hotmail.com)

$\dagger$ Investigador contribuyendo como primer autor.
} 


\section{Introducción}

La constante transformación de la sociedad actual exige que el modelo de formación docente se sustente en presupuestos teóricos - prácticos complejos y completos, que hagan posible dar respuesta a las diferentes y crecientes demandas de la educación, la competencia, profesionalidad y la capacidad de entrega que se le exige al profesorado hace recaer sobre sus espaldas (y la de los responsables del proceso de formación) una gran responsabilidad (Delors, 1996). La formación de profesores representa la principal esperanza para aquellos que confiamos en las capacidades de los propios docentes para hacer propuestas de mejora educativa (Marcelo, 2002).

En México, la formación inicial de profesores de educación básica se ha realizado históricamente en las escuelas normales. De acuerdo a estadísticas del ciclo escolar 2014 2015, existen en el país 484 escuelas normales que ofertan 23 licenciaturas a 132205 alumnos (Instituto Nacional de Evaluación [INEE], 2015). El informe Los docentes en México hace énfasis en la importancia del papel del profesor en el logro de aprendizajes de los estudiantes, por lo que resalta la necesidad de tener una formación inicial de calidad, por lo que recomienda que la aplicación del modelo educativo de las escuelas normales (de implementación reciente, en 2012) tenga correspondencia con los perfiles, parámetros e indicadores de ingreso y desempeño en la profesión docente, entre otras.

El 20 de agosto de 2012, con la publicación del Acuerdo 649 por el que se establece el Plan de estudios para la formación de maestros de educación primaria en el Diario Oficial de la Federación [DOF] y el inicio de su aplicación a nivel nacional en todas las escuelas normales del país, comienza una nueva etapa en la formación inicial docente (DOF, 2012).

El Plan de estudios 2012 para la Formación de Maestros de Educación Primaria tiene tres premisas principales: se centra en el aprendizaje del estudiante, posee flexibilidad administrativa y curricular y busca el desarrollo de competencias en cinco áreas principales: (1) preparación para la enseñanza y el aprendizaje, (2) psicopedagogía, (3) tecnologías para la comunicación e información e inglés, (4) arte, cultura y salud, en forma de materias optativas y (5) práctica profesional.
Aprender a ser profesor implica desarrollar la propia identidad como docente, por lo que en la formación inicial es necesario adoptar metodologías prácticas que se centren en explicar las causas y la influencia de tres aspectos: el profesor, la escuela y la actividad de aprendizaje (Pedder, 2011).

Una de las principales dificultades experimentadas durante la formación inicial como docente es relacionar las teorías que analizan en los estudios y lo que realmente pasa en la práctica docente. Los jóvenes expresan que lo que les enseñan sus mentores es en realidad diferente a lo que viven en el día a día. En el modelo educativo propuesto por el Plan de estudios 2012, el acercamiento a la práctica en condiciones reales de trabajo de los estudiantes se da en diferentes momentos en su carrera en los espacios programados para ello en los cursos del trayecto formativo de Práctica profesional (DGESPE, 2012a). Se entiende a la práctica profesional como el conjunto de acciones, estrategias e intenciones que un sujeto pone en juego para intervenir y transformar su realidad. En tanto actividad y acción de los profesionales de la educación, la práctica se concreta en contextos específicos, los cuales, brindan la posibilidad de lograr nuevos aprendizajes, de ahí que se reconozca el sentido formativo que tiene en el proceso de formación inicial de los futuros docentes (DGESPE, 2012a).

Desde esta perspectiva, el enfoque del plan de estudios reconoce que la práctica permite establecer una relación de ida y vuelta entre la teoría y la realidad, que se asocia más a las experiencias adquiridas en la escuela, en el caso de los docentes (Van der Berg, Rous y Beijard, 2014).

Desde el primer semestre, el acercamiento a las escuelas primarias es gradual, a partir del curso Observación y análisis de la práctica educativa. En segundo semestre, las actividades del curso Observación y análisis de la práctica escolar incluyen observaciones in situ en al menos tres instituciones diferentes, en distintos contextos. 
A partir del trabajo en el tercer semestre, el acompañamiento de los profesores de la escuela normal a los estudiantes es metodológico, teórico y didáctico, por lo que el trabajo del formador deberá centrarse en apoyar al estudiante, diseñando estrategias e instrumentos diferenciados que permitan recuperar evidencias de su propia práctica; debido a que uno de los aspectos fundamentales durante estos semestres es propiciar un acercamiento que contribuya a mejorar la docencia, además de la observación y la entrevista se agregan otro tipo de técnicas e instrumentos, como el diario, el portafolio de evidencias y la videograbación, entre otras (DGESPE, 2012a).

De este modo el trabajo del formador contribuirá a que el estudiante normalista en su etapa de formación inicial recupere sus experiencias y construya diferentes estrategias que permitan mejorar su docencia de manera permanente. Otra de las tareas del formador será la de enseñar a reflexionar, a analizar la propia docencia, lo que implica desarrollar en el estudiante normalista metahabilidades en las que la reflexión en la acción y la argumentación teórica se constituyen en acciones claves para la transformación de la docencia.

En este sentido, formar para la docencia implica asumir el reto donde la contradicción, la incertidumbre, las interacciones, los saberes, la certeza que da la experiencia acumulada será puesta a debate, para aspirar a formar un maestro nuevo y diferente (Mercado, 2007). El objetivo del siguiente trabajo es presentar los resultados obtenidos de la realización del proceso de diagnóstico propuesto como parte del curso Trabajo docente e innovación, que forma parte del $5^{\circ}$ semestre de la Licenciatura en Educación Básica. Este proceso generó conocimientos en dos sentidos: los conocimientos, habilidades y actitudes adquiridos por los docentes en formación así como la obtención de un panorama general del nivel inicial de los grupos de $5^{\circ}$ grado de primaria de la región norte de Nuevo León. Su estructura incluye una revisión de literatura relacionada con las competencias que se espera los docentes en formación alcancen en correspondencia a la posibilidad de organizar y evaluar el trabajo educativo y el desarrollo de la innovación en la formación inicial. Se explica también la conceptualización del diagnóstico que se construye a partir de la teoría y su aplicación en una situación real.
Se presenta en seguida el planteamiento del problema, originado en la revisión de la literatura y en la experiencia en la formación de docentes. En un tercer apartado se describe la metodología empleada en el análisis, así como los resultados obtenidos. Para finalizar se incluyen las conclusiones derivadas del estudio y las referencias bibliográficas.

\section{Revision de literatura}

Desde la perspectiva del modelo educativo en la escuela normal, las prácticas profesionales ofrecen la oportunidad de organizar comunidades de aprendizaje en las que se conjuga el conocimiento y experiencia del formador de la escuela normal, de los maestros de las escuelas primarias y del estudiante normalista.

Considerando el supuesto de que el diálogo, el debate y el análisis conjunto generan conocimiento (DGESPE, 2012).

En el transcurso en la formación inicial docente, hay diferentes momentos en los que se realizan prácticas en las escuelas primarias.

Estas actividades están en estrecha relación con el desarrollo de las competencias genéricas y profesionales incluidas en el perfil de egreso planteado por el Plan de estudios 2012. Las competencias genéricas se definen como las esperadas para un joven que termina la educación superior, mientras que las profesionales se relacionan de manera específica con ls exigencias actuales a la profesión docente e incluyen: (1) Diseña planeaciones didácticas, aplicando sus conocimientos pedagógicos y disciplinares para responder a las necesidades del contexto en el marco de los planes y programas de educación básica, (2) Genera ambientes formativos para propiciar la autonomía y promover el desarrollo de conocimientos, habilidades, actitudes y valores en los alumnos, (3) Aplica críticamente el plan y programas de estudio de la educación básica para alcanzar los propósitos educativos y contribuir al pleno desenvolvimiento de las capacidades de los alumnos del nivel escolar, (4) Usa las tecnologías de información y comunicación como herramienta de enseñanza y aprendizaje, (5) Emplea la evaluación para intervenir en los diferentes ámbitos y momentos de la tarea educativa. 
(6) Propicia y regula espacios de aprendizaje incluyentes para todos los alumnos, con el fin de promover la convivencia, el respeto y la aceptación, (7) Actúa de manera ética ante la diversidad de situaciones que se presentan en la práctica profesional, (8) Utiliza recursos de la investigación educativa para enriquecer la práctica docente, expresando su interés por la ciencia y por la propia investigación e (9) Interviene de manera colaborativa con la comunidad escolar, padres de familia, autoridades y docentes, en la toma de decisiones y en el desarrollo de alternativas de solución a problemáticas socioeducativas (DGESPE, 2012b).

\section{Trabajo docente e innovación}

Al llegar al cuarto semestre, los estudiantes normalistas han tenido diferentes experiencias en las escuelas primarias, lo que les ha permitido reconocer que la docencia es una acción compleja, en la que intervienen diferentes dimensiones (Fierro, Fortoul y Rosas, 2006).

El propósito del curso Trabajo docente e innovación es ofrecer elementos conceptuales y metodológicos para la innovación en la educación; además de contribuir al diseño de estrategias que contribuyan a la mejora de pa actuación docente; además potencia el uso de diagnósticos, seguimientos y evaluaciones, así como la experiencia de los estudiantes (DGESPE, 2014).

De manera particular se desarrollan y fortalecen las competencias profesionales 1, 3, 4, 5 y 8 , mencionadas en el apartado anterior, a partir de la situación problemática en la que se sitúa el curso, ¿es posible innovar y transformar de manera global la práctica docente? Carbonell (2007) define a la innovación como el resultado de una serie de intervenciones y procesos, con un propósito y sistematización, de manera que se modifiquen actitudes, ideas, culturas, contenidos, modelos y prácticas pedagógicas.

Para entender y aplicar la innovación en el aula, se guía a los estudiantes en dos etapas: la primera en la que se estudia, de manera teórica y práctica el concepto de diagnóstico en educación y se trabaja, en las escuelas primarias, a partir de la focalización y el diagnóstico para identificar aspectos que pueden mejorarse en la práctica docente.
En la segunda etapa, a partir del trabajo de diagnóstico se diseña e implementa un proyecto de innovación, con la finalidad de que el estudiante ponga en práctica una propuesta de innovación en el aula de clase, con el proósito de mejorar la enseñanza y el aprendizaje.

El diagnóstico en educación constituye un proceso de investigación con características científicas que pretende llegar al conocimiento de una situación, con la finalidad de actuar en si misma (Marí, 2007).

En relación con la innovación, Moreno (2000) explica que es un proceso en el que se involucran personas, situaciones e instituciones que interactúan en un tiempo y espacio determinados, implica transformaciones de las prácticas a través de la intervención, implica el diseño de acciones orientadas, articuladas, que se sustenten en el conocimiento sobre el objeto de innovación, a partir de los resultados de investigación.

\section{Perfil, parámetros e indicadores para docentes}

Una de las exigencias a la formación inicial docente en las escuelas normales es la relación entre la formación ofertada en ellas y las competencias que requieren los profesores de educación primaria en México (INEE, 2015). Estas características están expresadas en el documento Perfil, parámetros e indicadores para docentes y técnicos docentes (INEE, 2015).

El perfil del docente de educación primaria se organiza a partir de cinco dimensiones: (1) Un docente que conoce a sus alumnos, sabe cómo aprenden y lo que deben saber; (2) Un docente que organiza y evalúa el trabajo educativo, y realiza una intervención didáctica pertinente. (3) Un docente que se reconoce como profesional que mejora continuamente para apoyar a los alumnos en su aprendizaje; (4) Un docente que asume las responsabilidades legales y éticas inherentes a su profesión para el bienestar de los alumnos y (5) Un docente que participa en el funcionamiento eficaz de la escuela y fomenta su vínculo con la comunidad para asegurar que todos los alumnos concluyan con éxito su escolaridad (INEE, 2015). 
Estas dimensiones, se dividen en parámetros y éstos se subdividen en indicadores. Aprender la metodología para realizar un diagnóstico, diseñar un proyecto e implementarlo en el aula se relaciona de manera específica con el parámetro 5.1 Distingue los factores asociados a la gestión escolar que contribuyen a la calidad de los resultados educativos, y el indicador 5.1.1 Identifica los elementos básicos para realizar diagnósticos de los problemas que afectan los resultados educativos, el trabajo en el aula, la organización y el funcionamiento de la escuela y la relación con las familias (INEE, 2015, p. 38). Además, se contribuye al indicador 3.2.2 Identifica acciones para incorporar nuevos conocimientos $y$ experiencias al acervo con que cuenta y traducirlos en estrategias de enseñanza de la dimensión 3.2 Considera al estudio y al aprendizaje profesional como medios para la mejora de la práctica educativa (INEE, 2015, p. 36).

\section{La reflexión sobre la práctica}

El proceso de formación de los profesionales docentes requiere de propiciar la reflexión en, sobre y para la práctica, en las situaciones concretas y complejas que enfrentan por lo que es necesario que revisen y cuestionen las mismas imágenes, ideas y prácticas que han adquirido a lo largo de su vida personal y como estudiantes $\mathrm{y}$ que activan y practican en su quehacer cotidiano (Pérez, 2010).

Fernández, Rodríguez y Rodríguez (2010) proponen la consolidación de un modelo de práctico reflexivo que implica: (1) la inclusión de situaciones prácticas en el currículo, (2) el desarrollo de capacidades como apertura a los otros, la observación a una situación profesional desde distintos puntos de vista, y (3) el establecimiento de procesos de reflexión en y sobre la acción; poniendo en práctica estrategias como la investigación - acción, estudios de caso, aprendizaje basado en problemas, método de proyectos, uso de herramientas biográfico narrativas, entre otras.
Para Schön (1992), la formación en y para la práctica reside en el aprender haciendo, la reflexión sobre la acción y la buena tutoría. Schön (1992) afirma que los estudiantes aprenden a través de las prácticas aquello en lo que buscan convertirse en expertos si cuentan con el apoyo de otros veteranos, que los inician en las tradiciones de la práctica. Aprender haciendo es una manera de iniciación disciplinada al planteamiento y resolución de problemas de producción y actuación. La reflexión, es un proceso de encuadre, exploración y estructuración de problemas dentro de un contexto profesional determinado.

Este aprendizaje a partir de la reflexión sobre la acción requiere de un diálogo entre docente - experto y estudiante - novato, que se caracteriza por tener lugar en el contexto de los intentos del estudiante por intervenir en una situación real y concreta, utilizar lo mismo acciones y palabras y depender de una reflexión en la acción recíproca (Schön, 1992). En el caso de la formación inicial docente, las prácticas proporcionan experiencias diversas, constituyen espacios de confrontación, síntesis, análisis y crítica: la experiencia de estar, aunque sea en períodos cortos de tiempo en la realidad de la escuela primaria hace que los estudiantes normalistas distingan entre ser maestro $\mathrm{y}$ estudiante (Mercado, 2003).

En esta oportunidad, los estudiantes no solamente aprenden acerca del trabajo docente, sino que desarrollan habilidades para diagnosticar, investigar y analizar su práctica y los resultados educativos.

\section{Planteamiento del problema}

Díaz (2010) explica que cuando los docentes se enfrentan a la tarea de innovar, hay varios factores implicados. La aplicación del Plan de estudios 2012 para la formación de profesores ha generado, tanto en los docentes como en los estudiantes, nuevas dinámicas. En este estudio se indaga acerca de los conocimientos generados al aplicar un proceso de diagnóstico en un grupo de escuela primaria, durante la primera jornada de práctica docente del $5^{\circ}$ semestre, en el marco del curso Trabajo docente e innovación a partir de la pregunta: ¿Qué conocimientos se generan cuando los estudiantes normalistas de $5^{\circ}$ semestre realizan un proceso de diagnóstico educativo durante la jornada de práctica docente? 


\section{Metodología}

El estudio tiene un enfoque cualitativo, en el que se busca entender los fenómenos sociales desde el punto de vista de los actos, lo que importa es lo que las personas perciben como importante (Taylor y Bogdan, 1987). Se busca producir datos descriptivos, a partir de las propias palabras de los estudiantes normalistas y su conducta observable.

Los participantes son 24 estudiantes de $5^{\circ}$ semestre de la Licenciatura en Educación Primaria, Plan de estudios 2012 en una escuela normal pública, ubicada en el norte de México.

La realización del diagnóstico e informe se realizó en los meses de septiembre y octubre de 2014.

El método de recolección de información principal fue el análisis de documentos, en este caso se analizaron los informes de aplicación del diagnóstico en un grupo de educación primaria, para lo que se solicitó autorización a los participantes y se realizó un registro con la información de cada documento.

El análisis de los datos se realizó en etapas diferenciadas: (1) identificación de temas y desarrollo de conceptos y proposiciones, (2) codificación de datos y refinamiento de la comprensión del tema de estudio y (3) relativización de descubrimientos, comprendiendo los datos a partir del contexto en que fueron recopilados.

El énfasis del análisis se centró en dos apartados de cada informe: Resultados y Conclusiones. Estos apartados incluyen información cuantitativa y cualitativa. Los datos numéricos obtenidos, referidos la valoración que los estudiantes realizaron de dos aspectos (competencia lectora y resolución de problemas) se organizaron en una base de datos y se analizaron en función de estadística descriptiva. Los datos descriptivos (situación inicial del grupo, características de los niños, ambiente de trabajo) y las conclusiones se capturaron y analizaron, leyéndose en repetidas ocasiones para detectar semejanzas y diferencias, establecer categorías, contextualizar la información y generar conclusiones.

\section{Resultados}

\section{Estructura de los informes}

Al realizar el análisis inicial de los informes se detectó que su estructura incluyó los siguientes apartados: (1) Introducción; (2) Método (participantes, instrumentos, procedimiento, análisis). (3) Resultados (situación inicial del grupo, características de los niños, ambiente de trabajo, competencia lectora y resolución de problemas matemáticos); (4) Conclusiones; (5) Referencias bibliográficas y (6) Anexos. Todos los informes analizados corresponden a procesos de diagnóstico realizados en grupos de $5^{\circ}$ grado, del 08 al 19 de septiembre de 2014. La fecha de entrega del informe es 30 de septiembre de 2014.

En el apartado Introducción, los estudiantes mencionan qué es la evaluación diagnóstica, cuáles son los componentes del proceso de diagnóstico y explican lo que los niños deben saber a partir de los aprendizajes esperados de $4^{\circ}$ grado de educación primaria. Además, presentan la justificación de su trabajo en función de la importancia de conocer cinco aspectos del grupo con el que se empieza a trabajar: situación inicial, características de los niños, ambiente de trabajo, competencia lectora y habilidad para resolver problemas. Cada uno de estos aspectos se presenta con una definición con sustento teórico.

En Método, los informes reportan los participantes (600 alumnos de $5^{\circ}$ grado de 23 de escuelas primarias ubicadas en tres municipios). Los instrumentos empleados en el diagnóstico: entrevista al profesor, rejilla de observación, anecdotario, ejercicio para calcular lectura por minuto, ejercicio para valorar fluidez lectora, ejercicio para evaluar comprensión lectora, ejercicio para revisar el uso de algoritmos, ejercicio prediseñado de problemas matemáticos. En el procedimiento, se expone la temporalidad del período de recolección de datos (08 a 19 de septiembre, 2014) y se describe como se aplicó cada uno de los instrumentos (en la sección Apéndices, se incluyen evidencias de aplicación). 
En el apartado Análisis, se explica que los instrumentos se construyeron en colectivo, con asesoría del docente del curso Trabajo docente e innovación, asimismo, éste otorgó indicaciones y recomendaciones para su aplicación y proceso de análisis. La estrategia general de análisis fue cualitativa e incluyó varias etapas: (1) llenado de información en registros y concentrados, (2) lectura general de lo registrado, (3) identificación de la información reiterada mediante conteo de frecuencias, (4) organización de la información en tablas y gráficas y (5) Descripción de resultados.

En Resultados, la información se organiza en los apartados: (1) situación inicial del grupo, (2) características de los niños, (3) trabajo cotidiano, (4) competencia lectora y (5) resolución de problemas matemáticos. En estos apartados se presentan en descripciones (apartados 1, 2, 3), y también se complementan con tablas y gráficas (4 y 5).

En Conclusiones, los estudiantes explican su conceptualización de la evaluación diagnóstica, su valoración del proceso realizado, además de las fortalezas y áreas de oportunidad detectados en cada aspecto abordado. Focalizan, además el área de oportunidad que requiere mayor atención y la fortaleza del grupo que podrían emplear para atenderla. Los informes incluyen un mínimo de 7 referencias bibliográficas y un máximo de 12 , detectándose el uso de bibliografía que recomienda el curso Trabajo docente e innovación. En Apéndices se presentan las indicaciones que se siguieron para revisar y valorar la información de los instrumentos, la transcripción de entrevista al profesor, la rejilla de observación, el anecdotario, el registro de evaluaciones relacionadas con competencia lectora, el registro de evaluaciones de resolución de problemas matemáticos y ejemplos de ejercicios resueltos por los niños.

En los informes, se aprecia estructura y se presentan de manera formal, con un formato uniforme de acuerdo con las recomendaciones del Manual de Publicaciones de la American Psychological Association.

\section{Un panorama general de la situación de los grupos de $5^{\circ}$ grado en septiembre de 2014}

A partir de la revisión de los informes de los estudiantes es posible establecer un diagnóstico general de la situación inicial de los grupos de $5^{\circ}$ grado en el ciclo escolar 2014 - 2015. Las descripciones de los estudiantes en relación a la situación inicial del grupo desde el punto de vista de los profesores, las características de los niños y el ambiente de trabajo son diversas, los estdiantes normalistas describen la influencia del contexto en los comportamientos de los niños, así como algunas características comunes, originadas en la edad de los alumnos.

En la situación inicial del grupo desde la valoración de los profesores, se detectó la aplicación de un examen de diagnóstico de Español y Matemáticas en todos los casos, así como bajos resultados en ellos. En relación con las características de los niños, en general, se menciona interés por aprender, participación activa y también tendencia al descontrol y la indisciplina.

En ambiente de grupo, las descripciones de los estudiantes destacan la disciplina como factor para aprender, mencionan que los niños se llevan bien entre ellos, pero mencionan casos de violencia escolar y discriminación. En uno de los casos se habla de miedo a participar y compartir opiniones en clase.

En lo que se refiere a competencia lectora, los estudiantes analizaron tres aspectos: velocidad, fluidez y comprensión.

En velocidad, los resultados indican que el $35 \%$ de los niños de $5^{\circ}$ grado se ubican en el nivel Requiere apoyo, $16 \%$ Se acerca al estándar, $16 \%$ de ubica en el Estándar y 36\% de los niños demuestran un nivel de velocidad Avanzado.

Respecto a la fluidez, el 20\% de los niños de $5^{\circ}$ grado se ubican en el nivel Requiere apoyo, $24 \%$ Se acerca al estándar, 36\% de ubica en el Estándar y 24\% de los niños demuestran un nivel de velocidad Avanzado. 
En el ejercicio de comprensión, en el que se incluyeron 5 reactivos a los que se les otorgó un valor de dos puntos a cada uno, 92 niños obtuvieron la calificación máxima de 10, 128 calificó con 8,110 obtuvieron 6,89 tuvieron una calificación de 4, 61 niños solo registraron una respuesta correcta, sacando 2 y 50 niños sacaron 0 puntos, lo que indica el 17\%, 24\%, 21\%, 17\%, $12 \%$ y $9 \%$ de los estudiantes ubicados en cada calificación.

Para conocer la situación inicial de la habilidad para resolver problemas matemáticos, los jóvenes consideraron tanto algoritmos como problemas en los que se apliquen. En el ejercicio aplicado en todos los grupos, que consistió en cinco operaciones básicas obtenidas de una de las últimas lecciones del libro de texto de matemáticas de $4^{\circ}$ grado, la calificación promedio de grupo más alta fue de 9.38 , y la más baja de 0.17 , lo que indica una gran diversidad. El promedio general de los 27 grupos fue de 3.82. En 6 grupos se encontró que la operación con mayor dificultad era la división, en 5 la multiplicación y en 1 grupo ambas. En 7 grupos se presenta la principal dificultad en el algoritmo de la suma y en 1 ésta y la multiplicación. En 3 grupos el problema principal está en la resta. Un estudiante encontró que, en su grupo, todos los algoritmos presentan dificultades (lo que coincide con el grupo del promedio más bajo).

Al revisar los errores más comunes de los niños al resolver los algoritmos, en 9 de los grupos tienen que ver con acomodar las cifras, en 5 con no saber las tablas de multiplicación, en 4 grupos los niños tienen problemas de confusión de los signos $(+,-, \mathrm{x}, /)$; igual cantidad (4) tiene problemas al acomodar números decimales para hacer una operación; 3 grupos tienen como dificultad principal que los niños no cuentan bien y no dominan el procedimiento para resolver el algoritmo y en 2 grupos la principal dificultad reportada es que los niños requieren mayor tiempo para contestar correctamente.

En la habilidad de resolver problemas, los resultados indican una situación similar a la de las operaciones básicas: una gran diversidad en los niveles de aprovechamiento: la calificación promedio por grupo más alta es de 5.69 y la más baja de 0.7 , en una escala de 0 a 10. El promedio general es de 4.56 .
En las conclusiones del informe, cada estudiante normalista precisa el área de oportunidad, que a partir de su trabajo con los niños y la aplicación de los instrumentos de diagnóstico considera prioritaria. Las áreas de oportunidad prioritaria, organizadas de manera jerárquica de acuerdo con las frecuencias son: competencia lectora (11); habilidad para resolver problemas matemáticos (10); ambiente de aprendizaje (1), disciplina (1) y en un grupo se reporta tanto competencia lectora como habilidad matemática.

\section{Los aprendizajes de los estudiantes normalistas}

Al leer los apartados de conclusiones, se destaca que los estudiantes tienen una conceptualización propia del proceso de diagnóstico, que se origina tanto en la bibliografía revisada como en su experiencia práctica, tal y como lo registra la estudiante 14:

"La evaluación diagnóstica, desde mi perspectiva como docente en formación, sirve para conocer el nivel en que llegan los alumnos al pasar a otro grado y con los resultados, saber cuáles son sus fortalezas y áreas de oportunidad, para adecuar nuestras actividades y así poder ayudarles en lo que necesiten, además es importante conocer las características de los niños para lograr un ambiente de trabajo donde ellos puedan obtener los aprendizajes.

El proceso de evaluación diagnóstica realizado fue de gran utilidad para conocer los conocimientos que tienen actualmente los niños en las materias de español y matemáticas, y así saber como puedo trabajar con ellos de mejor manera".

Cada uno de los informes cierra con un apartado en el que los estudiantes seleccionan el área de oportunidad prioritaria y redactan de qué manera podrían atenderla, lo que sienta el precedente para el diseño de un proyecto de intervención didáctica, lo que implica que no solamente el reconocimiento del concepto de innovación como parte de la dinámica del trabajo docente, si no el desarrollo de ideas para su aplicación práctica en el siguiente período de trabajo en la escuela primaria. 


\section{Conclusiones}

En este estudio se presenta en una primera parte las características de la formación inicial de profesores y los indicadores de actuación que requiere el profesional de la educación actual. Además, se hace un recuento de las características del curso Trabajo docente e innovación, que gira en torno al desarrollo de los conceptos de diagnóstico e innovación en los futuros docentes, desde una perspectiva que busca el desarrollo de competencias y no solamente la adquisición teórica de definiciones.

Para lograr cumplir tanto con las competencias del perfil de egreso del Plan de estudios 2012 y el desarrollo de los componentes del Perfil, parámetros e indicadores para docentes de educación básica (INEE, 2015) es necesario que las actividades realizadas en la formación inicial consideren el desarrollo de bases teóricas, metodológicas y actitudinales que solamente se generan a partir de la aplicación de los conocimentos y habilidades adquiridas en el estudio de los cursos de la escuela normal en las actividades de práctica profesional realizadas en las escuelas de educación básica.

Si bien es cierto que la práctica hace al maestro, el desarrollo de competencias requiere ir más allá: implica que la realización de las actividades se formalice y sistematicen, considerando el rigor de la investigación científica, cosa que solo puede lograrse con la orientación y el compromiso de los académicos de la escuela normal.

El reporte Los docentes en México (INEE, 2015) argumenta que la formación que ofrecen las escuelas normales deja mucho que desear y no prepara realmente para la docencia. En este documento se recomienda, entre otras cosas, que entre sus actividades sustantivas se incluya la investigación y el desarrollo de intervenciones educativas, sobre todo para la enseñanza y el aprendizaje en la educación básica, y que guarden una estrecha relación de formación y de investigación con las escuelas en las que los alumnos ejercen sus prácticas, de manera en que las normales se conviertan en verdaderos laboratorios de enseñanza y se recupere lo mejor de la tradición normalista mexicana.
Como se aprecia en los resultados, este estudio es un intento de cumplir esta recomendación, además de contribuir al desarrollo de competencias de los participantes.

La sistematización de la aplicación del diagnóstico y el análisis de sus resultados permite, además de recuperar los aprendizajes de los estudiantes normalistas, proporciona un panorama general de las características de los grupos de las escuelas primarias en las que los estudiantes laboraron, esta información no solamente es útil para ellos, si no también para los docentes y directivos de las escuelas, asesores técnico pedagógicos y supervisores; los profesores de la escuela normal e incluso para las autoridades educativas.

Lo que se requiere es la voluntad de aprender junto con los estudiantes la utilidad de la aplicación de la metodología cualitativa para analizar los procesos educativos, utilizando enfoques de reflexión sobre la práctica e investigación - acción. Esta experiencia, es solamente el punto de partida: a partir de los resultados obtenidos en su diagnóstico, se espera que cada estudiante diseñe y aplique un proyecto de innovación, que mejore su práctica docente y atienda el área de oportunidad detectada.

La generación de conocimientos sobre los niños, las escuelas primarias, las prácticas docentes, los aprendizajes de los docentes en formación, las implicaciones para los formadores, las diferentes aristas del reto de la transformación de las escuelas normales a partir de la aplicación de un nuevo modelo educativo, en el que la investigación educativa tiene un papel importante como estrategia para la mejora, abre la posibilidad de iniciar una nueva etapa en la historia de la formación docente en México.

\section{Referencias}

Delors, J. (1996). La educación encierra un tesoro Informe a la UNESCO de la Comisión Internacional sobre la educación para el Siglo XXI. España: Santillana - UNESCO.

Diario Oficial de la Federación (DOF). (2012). Acuerdo 649 por el que se establece el Plan de estudios para la formación de maestros de educación primaria. México: Autor. 
Díaz, F. (2010). Los profesores ante las innovaciones curriculares. Revista Iberoamericana de Educación Superior RIES 1 (1), $37-57$.

Dirección de Educación Superior para Profesionales de la Educación (DGESPE). (2012a). El trayecto de Práctica profesional: Orientaciones para su desarrollo. México: DGESPE.

Dirección de Educación Superior para Profesionales de la Educación (DGESPE). (2012b). Plan de estudios 2012 para la Formación de Maestros de Educación Primaria. México: DGESPE.

Dirección de Educación Superior para Profesionales de la Educación (DGESPE). (2013). Trabajo docente e innovación. México: DGESPE.

Fernández, E., Rodríguez, H. y Rodríguez, M. (2010). La formación inicial de profesionales de la educación: un análisis crítico de los nuevos planes de estudio en el contexto del capitalismo académico. Revista Interuniversitaria de Formación del Profesorado 24 (2) 151 - 174.

Fierro, C., Fortoul, B. y Rosas, L. (2008). Transformando la práctica docente Una propuesta basada en la investigación - acción. ( $1^{\mathrm{a}}$ reimpresión). México: Paidos.

Instituto Nacional de Evaluación para la Educación [INEE]. (2015). Perfil, parámetros e indicadores para docentes y técnicos docentes. México: INEE.

Instituto Nacional de Evaluación para la Educación [INEE]. (2015). Los docentes en México Informe 2015. México: INEE.

Marcelo, C. (2002). La formación inicial y permanente de los educadores. En Consejo Escolar del Estado. Los educadores en la sociedad del siglo XXI. España: Ministerio de Educación, Cultura y Deporte. pp. 161 - 194.

Marí, R. (2007). Diagnóstico pedagógico Un modelo para la intervención psicopedagógica. (2a ed.). España: Ariel.
Mercado, E. (2003). De estudiante a maestro practicante: los ritos de paso en las prácticas pedagógicas en la escuela normal. Tiempo de educar 4 (7) $121-151$.

Mercado, E. (2007). Formar para la docencia. Una aproximación al trabajo de los asesores y tutores en la escuela normal. Revista Mexicana de Investigación Educativa (12)487 - 512.

Moreno, M. (2000). Formación docente para la innovación educativa. Sinéctica (7) $24-32$. Pedder, V. D. (2011). Conceptualizing teacher profesional learning. Review of Educational Research 81(3), 376 - 407.

DOI: $10.3102 / 0034654311413609$

Pérez, A. (2010). Nuevas exigencias y escenarios para la profesión docente en la era de la información y de la incertidumbre. Revista Interuniversitaria de Formación del Profesorado, 24 (2) $17-36$.

Schön, D. (1992). La formación de profesionales reflexivos: Hacia un nuevo diseño de la enseñanza y el aprendizaje de las profesiones. España: Paidós.

Taylor, S. y Bogdan, R. (1987). Introducción a los métodos cualitativos de investigación: la búsqueda de significados. México: Paidós.

Van del Bergh, L., Ros, A. y Beijaard, D. (2014). Improving teacher feedback during active learning: effects of a professional development program. American Educational Research Journal 20(10), 1 - 37. DOI: $10.3102 / 0002831214531322$ 